\title{
Estimating the Taylor Rule in the Time-Frequency
}

\section{Domain $^{* \dagger}$}

\author{
Luís Aguiar-Conraria ${ }^{\ddagger}$ Manuel M. F. Martins ${ }^{\S} \quad$ Maria Joana Soares $₫$ \\ May 18, 2018
}

\begin{abstract}
We present the first assessment of U.S. monetary policy across time and frequencies within the Taylor Rule framework. We derive a novel wavelet tool - the partial wavelet gain to estimate a parametric equation relating the federal funds rate to inflation and the output gap. We detect a gradual shift of the focus of policy from short cycles to intermediate cycles at the beginning of the Great Moderation, followed by a strengthening of policy's reaction to long fluctuations once credibility was attained, and, during the Great Recession, a renewed interest in shorter output cycles. We document that the violation of the Taylor principle until the early 1980s and the strengthening of the reaction of policy to inflation thereafter were more marked at intermediate than at long cycles. Overall, we also detect lead-lag relationships between the policy rate and inflation and the output gap that differ along time and cyclical frequencies.
\end{abstract}

Keywords: Monetary Policy; Taylor Rule; Partial Wavelet Gain; Time-Frequency Estimation; Continuous Wavelet Transform.

*We thank Rita Carreira, two anonymous referees and the editor for very important comments that vastly improved the quality of our work. The usual disclaimer applies.

†The data and MatLab scripts necessary to replicate all our results are available for download at http://sites.google.com/site/aguiarconraria/joanasoares-wavelets. In the same website, the reader can find and freely download a wavelet MatLab toolbox that we wrote.

${ }^{\ddagger}$ NIPE and Department of Economics, University of Minho, E-mail address: lfaguiar@eeg.uminho.pt. Corresponding author.

$\S$ Cef.up and Faculty of Economics, University of Porto, E-mail address: mmfmartins@fep.up.pt

"NIPE and Department of Mathematics and Applications, University of Minho, E-mail address: jsoares@math.uminho.pt 
JEL codes: C49, E43, E52.

\section{Acknowledgments}

Research at NIPE was carried out within the funding with COMPETE reference number POCI01-0145-FEDER-006683 (UID/ECO/03182/2013), with the FCT/MEC's (Fundação para a Ciência e a Tecnologia, I.P.) financial support through national funding and by the ERDF through the Operational Programme on "Competitiveness and Internationalization - COMPETE 2020 under the PT2020 Partnership Agreement.

Research at Cef.up has been financed by Portuguese Public Funds through FCT (Fundação para a Ciência e a Tecnologia) and by the European Regional Development Fund through COMPETE 2020 - Programa Operacional Competitividade e Internacionalização - in the framework of the project UID/ECO/04105/2013. 


\section{Introduction}

This paper uses continuous wavelet tools to estimate the coefficients of the Taylor Rule that are implicit in U.S. monetary policy between 1965 and 2017. The simultaneous variation of coefficients along time and frequencies and the thorough statistical analysis provided by our tools allow for detecting new stylized facts about the last five decades of U.S. monetary policy.

Taylor (1993) showed that, between 1986 and 1992, monetary policy in the United States could be well described by a simple parametric relationship between the policy interest rate, the output gap, and inflation

$$
\mathrm{FFR}_{t}=2+\pi_{t}+\frac{1}{2} y_{t}+\frac{1}{2}\left(\pi_{t}-2\right)
$$

in which $\mathrm{FFR}_{t}$ is the (effective) federal funds rate in period $t, \pi_{t}$ is the inflation rate over the previous four quarters, $y_{t}$ is the percent deviation of output from its potential and both the real equilibrium interest rate and the inflation target are assumed to equal 2 percent. Thereafter, a great deal of models and analyses of U.S. monetary policy have focused on Taylor-type rules.

The Taylor Rule (TR) is important both from positive and normative perspectives. Some even argue that it should be used as a policy accountability device.

From a positive point of view, this approach is useful to describe, in a very parsimonious

way, U.S. monetary policy. Indeed, subsequent studies have shown that such broad empirical success extends to periods before 1986 and after 1992, which is particularly notable given that, as documented inter alia by Kahn (2012) and Taylor (2012), there were no references to Taylor-type rules in the Federal Open Market Committee meetings before 1993, and, even after 1993, the Federal Reserve never acknowledged adhering to a Taylor-type rule.

From a normative point of view, TR may also be considered a useful benchmark for monetary policy, highly valuable in informing and aiding policymakers' decisions, even if it is not to be followed mechanically. As an approximation to the optimal control solution of the monetary policymaker's problem, TR has been proven to be quasi-optimal and more robust than a wide array of strictly optimal policy rules derived in specific macroeconomic models — see e.g. Taylor and Williams (2010). Moreover, it has the advantage of simplicity, making it very easy to communicate and understand. 
The argument that, under the TR, monetary policy is more predictable, systematic, and effective (Taylor, 2012), has brought attention, in policy circles, to Taylor and Williams' (2010) suggestion that it could become an accountability device; there was even a bill introduced in the U.S. Congress, which fueled discussions in academic circles — see e.g. Bernanke (2015) and Walsh $(2015){ }^{1}$

A very large literature thoroughly documents how TR coefficients have changed over time, ${ }^{2}$ but is virtually silent on the intensity of the changes across different cyclical oscillations. There is a smaller literature that documents different TR coefficients across some cyclical frequencies, but limits time-domain considerations to a comparison between a very limited set of sub-sample periods. ${ }^{3}$ There are ample reasons for the TR coefficients to behave differently across frequencies. First, policymakers should care about the impact of policy across cyclical frequencies because oscillations at different frequencies may have different impacts on social welfare; also, controlling oscillations at some frequencies may imply a trade-off with larger variability at other frequencies (Yu, 2013). Second, while arguably policymakers react more strongly to persistent than to shortlived fluctuations in the main macroeconomic variables, the relative importance of controlling low versus medium versus high frequency oscillations may depend on the circumstances. For example, the discussion about which inflation rate to consider in the TR — whether headline inflation or core inflation, which features smaller high-frequency variation — is an example of the difficulty in finding a one-size-fits-all best indicator for policy (see e.g. Mehra and Sawhney, 2010). Third, monetary policy's relative reaction at different frequencies may depend on temporary factors; for example, a policymaker trying to conquer credibility may have to react very strongly to transitory changes in inflation, but once credibility is established, he or she may instead focus on fluctuations of a more permanent nature (Ashley, Tsang and Verbrugge, 2015).

\footnotetext{
${ }^{1}$ H.R. 5018 (113th) Federal Reserve Accountability and Transparency Act of 2014, discussed in the House Financial Service Committee, according to which the FED should explain to the House any systematic deviations of the policy interest rates from a reference policy interest rate that would correspond precisely to that implied by Taylor's (1993) Rule presented in (1). For details, see https://beta.congress.gov/113/bills/hr5018/BILLS-113hr5018ih.pdf

${ }^{2}$ E.g., following Clarida, Galí and Gertler's (2000) finding that U.S. interest rate policy has been more sensitive to inflation after 1979, the stability of the U.S. TR has been assessed using several time-series methods, such as threshold models (Bunzel and Wenders, 2010), time-varying parameters models (Trecroci and Vassalli, 2010), Markov-switching models (Assenmacher-Wesche, 2006), smooth-transition models (Alcidi, Flamini and Fracasso, 2011), instrumental variables quantile regressions (Wolters, 2012), and Hamilton's (2001) flexible approach to nonlinear inference (Kim, Osborn and Sensier, 2005).

${ }^{3}$ E.g. Ahmed, Levin and Wilson (2004), Pancrazi (2015) and Ashley, Tsnag and Verbrugge (2015)
} 
Hence the motivation for this paper: we use continuous wavelet tools, with an approach consisting of a sequential analysis of partial wavelet coherencies, phase-difference diagrams, and gains, which will allow us to study changes in the relationship between the policy interest rate and the macroeconomic variables included in TR across time and frequencies.

A first contribution of the paper is methodological, for we provide a multi-variable generalization of the wavelet gain (the partial wavelet gain), which allows us to estimate regression coefficients in the time-frequency domain. ${ }^{4}$ Moreover, we use the multiple coherency (see AguiarConraria and Soares, 2014) and the partial coherency, as well as the partial phase-difference, to refine the interpretation of the estimates given by the partial gain. Our paper is the first to employ such an integrated analysis of this set of continuous wavelet tools to effectively estimate a dynamic multivariate function in the continuous time-frequency space.

A second contribution is to provide a set of new stylized facts about the history of U.S. monetary policy, describing how the TR coefficients have changed over the last five decades, particularly at the cyclical frequency level, in a continuous-time framework. By providing estimates of the monetary policymaker's reaction to fluctuations in inflation and output over time and across frequencies, we offer insight on how the policymaker may have dealt with the trade-offs inherent to stabilizing different cycles, as well as how he/she may have adapted policy in response to changes in circumstances and policy regimes.

Before we proceed, a word on the specification of the TR used in this paper is warranted. While the literature features a number of variations of the TR, we focus on Taylor's original specification (1993) because (1) we want to benefit from the robustness of the baseline formulation, and (2) our approach endogenously deals with many of the uncertainties surrounding the specification of the TR - namely, whether it has been contemporaneous, forward- (as assumed in most analyses), or backward-looking. ${ }^{5}$

There are two caveats to our approach due to technical constraints: first, we do not account for interest rate smoothing, as the inclusion of the lag of the endogenous variable would soak up

\footnotetext{
${ }^{4}$ A filter gain is simply the ratio of the amplitude of the output over the amplitude of the input at a given frequency. As pointed out by Engle (1976), in spectral analysis, the Fourier gain between two variables can be interpreted as their regression coefficient at a given frequency. Mandler and Scharnagl (2014) apply this concept in wavelet analysis.

${ }^{5}$ Lee, Morley and Shields (2015) have also taken an agnostic approach to the TR specification.
} 
all the variation in the FFR; and, second, we cannot assess the time-frequency variation of the constant of the TR. Thus, neither do we assess a potentially varying inflation target, nor are we able to account for a potentially varying natural rate of interest - continuous wavelet tools imply looking at each frequency separately and, naturally, information about the constant is lost as it does not correspond to any frequency.

The paper proceeds as follows. In section 2, we describe our methodology for the case of three variables, presenting the key results and an illustrative example. In Section 3, we describe the data and further motivate our time-frequency approach. In Section 4, we apply wavelet tools to the data and provide a continuous time-frequency assessment of the U.S. TR. Section 5 concludes. In the appendix, we provide a self contained summary of our methodology, including a generalization of the partial wavelet gain for any number of variables.

\section{Methodology}

The continuous wavelet transform is an increasingly popular tool in econometric analysis. The most common argument to justify its use is the possibility of tracing transitional changes across time and frequencies. Economic data is most of the times noisy, strongly nonstationary, with possible nonlinear relations. The local nature of wavelet analysis makes it very suitable to use with this kind of data. See Aguiar-Conraria and Soares (2014) and the chapters included in Gallegati and Semmler (2014) for a review and recent developments. So far, the analysis in the time-frequency domain with the continuous wavelet transform has been mostly limited to the use of the wavelet power spectrum, the wavelet coherency and the wavelet phase-difference. AguiarConraria and Soares (2014) already extended these tools to allow for multivariate analyses, and such tools are sufficient to assess the strength of the relationship between several variables, but are insufficient to estimate the relationship's magnitude, much like (partial) correlation coefficients, which do not provide the same information as regression coefficients.

Mandler and Scharnagl (2014) use the concept of wavelet gain as a regression coefficient in the regression of $y$ on $x$. In this paper, and, to our knowledge, for the first time, we will estimate an equation relating more than two variables (similar to a regression of $y$ on several variables) in the 
time-frequency domain.

To do so, we generalize the concept of wavelet gain and define the partial wavelet gain, which can be interpreted as a regression coefficient in the regression of $y$ on $x$ after controlling for other variables. In this section, for ease of exposition, we will consider the special case of three variables. We leave for the appendix, as it demands the use of some cumbersome matrix notation, the general case applicable to any number of variables.

The reader who is not interested in the technical details may skip to subsection 2.6 , where we intuitively explain how to interpret the more innovative wavelet measures that we develop in this paper.

\subsection{The Continuous Wavelet Transform}

For all practical uses, a wavelet $\psi(t)$ is a function that oscillates around the $t$-axis and looses strength as it moves away from the center, behaving like a small wave. The specific wavelet we use in this paper is the complex-valued function (selected from the so-called Morlet wavelet family) defined by $\psi(t)=\pi^{-\frac{1}{4}} e^{6 i t} e^{-\frac{t^{2}}{2}}$. See e.g. Aguiar-Conraria and Soares (2014) for a discussion of some desirable properties of this wavelet, which make its use attractive. ${ }^{6}$

Given a time-series $x(t)$, its continuous wavelet transform (CWT), with respect to a given wavelet $\psi$, is the function of two variables, $W_{x}(\mathrm{t}, \mathrm{s})$, given by

$$
W_{x}(\mathrm{t}, \mathrm{s})=\frac{1}{\sqrt{|\mathbf{s}|}} \int_{-\infty}^{\infty} x(t) \bar{\psi}\left(\frac{t-\mathrm{t}}{\mathrm{s}}\right) d t
$$

Note that $\mathrm{s}$ is a scaling parameter and $\mathrm{t}$ a translation parameter controlling, respectively, the width and the location along the $t$-axis of the function $\psi\left(\frac{t-\mathrm{t}}{\mathrm{s}}\right)$; for $|\mathrm{s}|>1$, the function becomes larger (hence, corresponding to a function with lower frequency) and for $|\mathbf{s}|<1$, it becomes narrower (hence, becoming a function with higher frequency); in the above formula and throughout the paper, an over-bar denotes complex conjugation.

Remark 1 As for the CWT, all the wavelet measures that we are going to introduce are functions

\footnotetext{
${ }^{6}$ This is the most commonly used wavelet in Economics, when performing a continuous wavelet analysis; for robustness checks, other analytic wavelets from the Generalized Morse Wavelet family were used and gave similar results to the ones described in the paper.
} 
of the two variables, $\mathrm{t}$ and $\mathrm{s}$. To simplify the notation, we will describe these quantities for a specific value of the argument, (t, s), which will be omitted in the formulas.

\section{$2.2 \quad$ Uni and bivariate tools}

\subsubsection{Wavelet power spectrum and the phase angle}

Similarly to the terminology used in the Fourier case, the (local) wavelet power (spectrum) is defined as

$$
(W P S)_{x}=W_{x} \overline{W_{x}}=\left|W_{x}\right|^{2}
$$

The wavelet power spectrum gives us a measure of the variance distribution of the time-series in the time-frequency plane.

When the wavelet $\psi$ is complex-valued, as in our case, the wavelet transform $W_{x}$ is also complex-

valued. In this case, the transform can be expressed in polar form as $W_{x}=\left|W_{x}\right| e^{i \phi_{x}}, \phi_{x} \in(-\pi, \pi]$. The angle $\phi_{x}$ is referred to as the (wavelet) phase

\subsubsection{Cross wavelet tools}

The cross-wavelet transform of two time-series $y(t)$ and $x(t)$, denoted by $W_{y x}$, is defined as

$$
W_{y x}=W_{y} \overline{W_{x}}
$$

and its absolute value is referred to as the cross-wavelet power. The cross-wavelet power of two time-series depicts the covariance between two time-series at each time and frequency.

The complex wavelet coherency of $y$ and $x, \varrho_{y x}$, is given by

$$
\varrho_{y x}=\frac{S\left(W_{y x}\right)}{\left[S\left(\left|W_{y}\right|^{2}\right) S\left(\left|W_{y}\right|^{2}\right)\right]^{1 / 2}}
$$

where $S$ denotes a smoothing operator in both time and scale. For simplicity, we will denote by $S_{y x}$ the smoothed cross-wavelet transform of two series $y$ and $x$, i.e. $S_{y x}=S\left(W_{y x}\right)$; we will also use $\sigma_{x}$ to denote the square root of the smoothed wavelet power of series $x$, i.e., $\sigma_{x}=\sqrt{S\left(\left|W_{x}\right|^{2}\right)}=\sqrt{S_{x x}}$, 
and use similar notation for series $y$. Hence, the formula for the complex coherency can simply be written as:

$$
\varrho_{y x}=\frac{S_{y x}}{\sigma_{y} \sigma_{x}}
$$

The wavelet coherency, which we will denote by $R_{y x}$, is the absolute value of the complex wavelet coherency, i.e., $R_{y x}=\left|\varrho_{y x}\right|=\frac{\left|S_{y x}\right|}{\sigma_{y} \sigma_{x}}$.

With a complex-valued wavelet, we can compute the phase of the wavelet transform of each series and, by computing their difference, we can obtain information on the possible delays of the oscillations of the two series, as a function of time and frequency. It follows immediately from (4) that the (wavelet) phase-difference between $y$ and $x$, which we will denote by $\phi_{y x}$, can also be computed as the angle of the cross-wavelet transform, $W_{y x}$. Another slightly different way to define the phase-difference makes use of the angle of the complex wavelet coherency, instead of the angle of the cross-wavelet transform; this definition, although not strictly coinciding with the difference between the individual phases, due to smoothing, has the advantage of allowing a more direct generalization for the multivariate case. Finally, we define the complex wavelet gain of $y$ over $x$, denoted by $\mathcal{G}_{y x}$, as

$$
\mathcal{G}_{y x}=\frac{S_{y x}}{S_{x x}}=\varrho_{y x} \frac{\sigma_{y}}{\sigma_{x}}
$$

and, following Mandler and Scharnagl (2014), we call wavelet gain, and denote by $G_{y x}$, the modulus of the complex wavelet gain, i.e.,

$$
G_{y x}=\frac{\left|S_{y x}\right|}{S_{x x}}=R_{y x} \frac{\sigma_{y}}{\sigma_{x}} .
$$

Recalling the interpretation of the Fourier gain as the modulus of the regression coefficient of $y$ on $x$ at a given frequency (see, e.g. Engle 1976), it is perfectly natural to interpret the wavelet gain as the modulus of the regression coefficient in the regression of $y$ on $x$, at each time and frequency.

\subsection{Multivariate wavelet tools (case of three series)}

Here we present the formulas for the wavelet multivariate tools - multiple wavelet coherency, partial wavelet coherency, partial wavelet phase-difference, and partial wavelet gain — for the simplest case in which we only have three series $y, x$ and $z$; the formulas for the general case, from 
which the particular formulas here presented were obtained, are given in the appendix.

The squared multiple wavelet coherency between the series $y$ and the two series $x$ and $z$, denoted by $R_{y(x z)}^{2}$ is given by

$$
R_{y(x z)}^{2}=\frac{R_{y x}^{2}+R_{y z}^{2}-2 \Re\left(\varrho_{y x} \varrho_{x z} \overline{\varrho_{y z}}\right)}{1-R_{x z}^{2}}
$$

and the multiple wavelet coherency between the series $y$ and the series $x$ and $z$, denoted by $R_{y(x z)}$, is the positive square root of the above quantity.

The complex partial wavelet coherency between $y$ and $x$, after controlling for $z$, is given by

$$
\varrho_{y x . z}=\frac{\varrho_{y x}-\varrho_{y z} \overline{\varrho_{x z}}}{\sqrt{\left(1-R_{y z}^{2}\right)\left(1-R_{x z}^{2}\right)}} .
$$

The absolute value and the angle of $\varrho_{y x . z}$, will, respectively, be called the partial wavelet coherency and the partial (wavelet) phase-difference between the series $y$ and $x$, after controlling for $z$, and be denoted by $R_{y x . z}$ and $\phi_{y x . z}$.

The complex partial wavelet gain between series $y$ and $x$, after controlling for $z, \mathcal{G}_{y x . z}$, is given by

$$
\mathcal{G}_{y x . z}=\frac{\varrho_{y x}-\varrho_{y z} \overline{\varrho_{x z}}}{\left(1-R_{x z}^{2}\right)} \frac{\sigma_{y}}{\sigma_{x}}
$$

and its absolute value is called the partial wavelet gain and is denoted by $G_{y x . z}$, i.e.,

$$
G_{y x . z}=\frac{\left|\varrho_{y x}-\varrho_{y z} \overline{\varrho_{x z}}\right|}{\left(1-R_{x z}^{2}\right)} \frac{\sigma_{y}}{\sigma_{x}} .
$$

\subsection{Representation of wavelet measures and statistical significance}

Note that, as observed before, each of the above wavelet measures is a function of the two variables, $\mathrm{t}$ (time) and s (scale or frequency). In practice, all these wavelet measures are computed for a finite number of time and scale (frequency) values, producing a matrix, the information of which must be appropriately displayed for ease of interpretation.

The wavelet power and the wavelet coherencies are plotted as 2-dimensional heat-maps, with colors ranging from blue (indicating low power/small coherency) to red (high power/high coherency). 
When computing the CWT of a finite length time-series, the values of the transform at the beginning and the end of the series involve missing values, which are then artificially prescribed, causing inevitable distortions. The region in which the transform suffers from these edge effects and where, naturally, the results should always be interpreted carefully is called the cone-of-influence (COI). The identification of the COI in the plots of the wavelet power and wavelet coherencies is done by drawing an appropriate line.

To test the significance of the wavelet power spectrum, one can rely on the results of Torrence and Compo (1998), which show that the local wavelet power spectrum of a white noise or an AR(1) process, normalized by the variance of the time series, is quite well approximated by a chi-squared distribution. Testing the wavelet power spectrum against a flat spectrum (white noise) is a good starting point and is the one we used in this paper. ${ }^{7}$

There are no good theoretical results to test the significance of coherency and partial coherency, as the available ones impose restrictions that are too stringent. Therefore, one usually relies on Monte-Carlo simulations. In our case, we fit an $\operatorname{ARMA}(1,1)$ model to each of the series and construct new samples by drawing errors from a Gaussian distribution with a variance equal to that of the estimated error terms; for each set of time-series, we performed this exercise 5000 times, and then extracted the critical values. In the plots of the power and of the wavelet coherencies, the $5 \%$ and $10 \%$ significance levels are identified with a black and a gray contour, respectively.

For the phases (or phase-differences) and for the gains, we display mean values (at each point in time) corresponding to some selected frequency bands. Since the phases are angular measures, each mean phase is computed as a circular mean, which is the appropriate notion of mean in this case; see, e.g. Zar (1998). Confidence intervals for the circular mean at each point in time were also computed — we used the formulas proposed in Zar (1996), p. 604; see also Berens (2009) - and the interpretation of the mean phase at each point is done considering values as extreme as the two-end points of the corresponding interval. The limits of the confidence intervals for the mean phases are indicated in the pictures with black dashed-lines. Each mean gain in a given frequency band is obtained by computing the absolute value of the mean of the corresponding

\footnotetext{
${ }^{7}$ If one wants to consider more complicated null hypotheses, rather than a white or red noise, one usually relies on Monte-Carlo simulations.
} 
complex gains. ${ }^{8}$ How to appropriately obtain confidence intervals for the gain is a question which still remains open; for this reason, one should complement the analysis of the gain by inspecting coherency, and only focus on the regions whose corresponding coherency is statistically significant.

\subsection{Example: Partial coherency, partial phase-difference and partial gain}

We now give a constructed example illustrating the application of the partial wavelet gain proposed in this paper. Given full control of the data generating processes, our example makes it clear that the partial wavelet gain may be interpreted as a regression coefficient in the time-frequency domain. The example also highlights that, because the (partial) wavelet gain is an absolute value, its interpretation must be complemented with that of the (partial) wavelet phase-difference, which will tell us if the relationship is positive or negative and, at the same time, indicate which variable is leading.

The values for the (partial) phase-difference may be interpreted as follows: a (partial) phasedifference between $y$ and $x$ (after controlling for other variables) with value zero indicates that the time-series move together at the specified frequencies; if the (partial) phase-difference is between 0 and $\pi / 2$, then both series move in the phase, but $y$ leads $x$; if the (partial) phase-difference is between $-\frac{\pi}{2}$ and 0 , then it is $x$ that is leading; a (partial) phase-difference of $\pi$ indicates an anti-phase relationship; if the (partial) phase-difference is in $(\pi / 2, \pi)$, then $x$ is leading; time-series $y$ is leading, if the (partial) phase-difference lies in $\left(-\pi,-\frac{\pi}{2}\right)$.

Assume that we have monthly data and the data generating processes for $X$ and $Z$ are given by

$$
\begin{aligned}
& X_{t}=\sin \left(2 \pi \frac{t}{3}\right)+\sin \left(2 \pi \frac{t}{8}\right)+\varepsilon_{x, t}, \\
& Z_{t}=\sin \left(2 \pi \frac{t}{9}\right)+\varepsilon_{z, t}
\end{aligned}
$$

\footnotetext{
${ }^{8}$ An alternative would be to consider a weighted mean, where the weights would be related to the partial coherency value. Exploring this alternative is left for future research.
} 
while for $Y$ is given by

$$
Y_{t}=\left\{\begin{array}{ll}
2 \sin \left(2 \pi \frac{t+3 / 12}{3}\right)+1 \sin \left(2 \pi \frac{t-1}{8}\right)+Z_{t}+\varepsilon_{y, t}, & \text { for } t \leq 100 \\
2 \sin \left(2 \pi \frac{t+3 / 12}{3}\right)-3 \sin \left(2 \pi \frac{t-1}{8}\right)+Z_{t}+\varepsilon_{y, t}, & \text { for } t>100
\end{array} .\right.
$$

Suppose that we are interested in doing the equivalent of regressing $Y$ against $X$ in the timefrequency domain. What results should we expect?

At frequencies that correspond to a period of 3 years, the estimated coefficient should be 2 throughout the sample, implying that the wavelet gain should also be 2 . The phase-difference should also indicate that $Y$ slightly leads $X$ (by 3 months), meaning that the phase-difference between $Y$ and $X$ should be between 0 and $\pi / 2$. At the frequency corresponding to an 8-year period, the coefficient should be +1 in the first half of the sample and -3 in the second half. However, given that the wavelet gain is an absolute value, it would yield an estimate of +3 for the coefficient in the second half of the sample. To capture the negative sign of the relationship, one would have to use the information given by the phase-difference. In the first half of the sample, at this frequency, $Y$ lags $X$ (by 1 year) and the variables are in-phase; therefore, the phase difference should be between $-\pi / 2$ and 0 . In the second half, $Y$ lags $X$ (by 1 year) and the variables are out-of-phase; therefore, the phase-difference should be between $\pi / 2$ and $\pi$.

Finally, note the influence of $Z$ on variable $Y$ : given that its influence occurs at a frequency corresponding to a 9-year period, excluding this variable should contaminate the relationship between $Y$ and $X$ at the 8-year period frequency. 

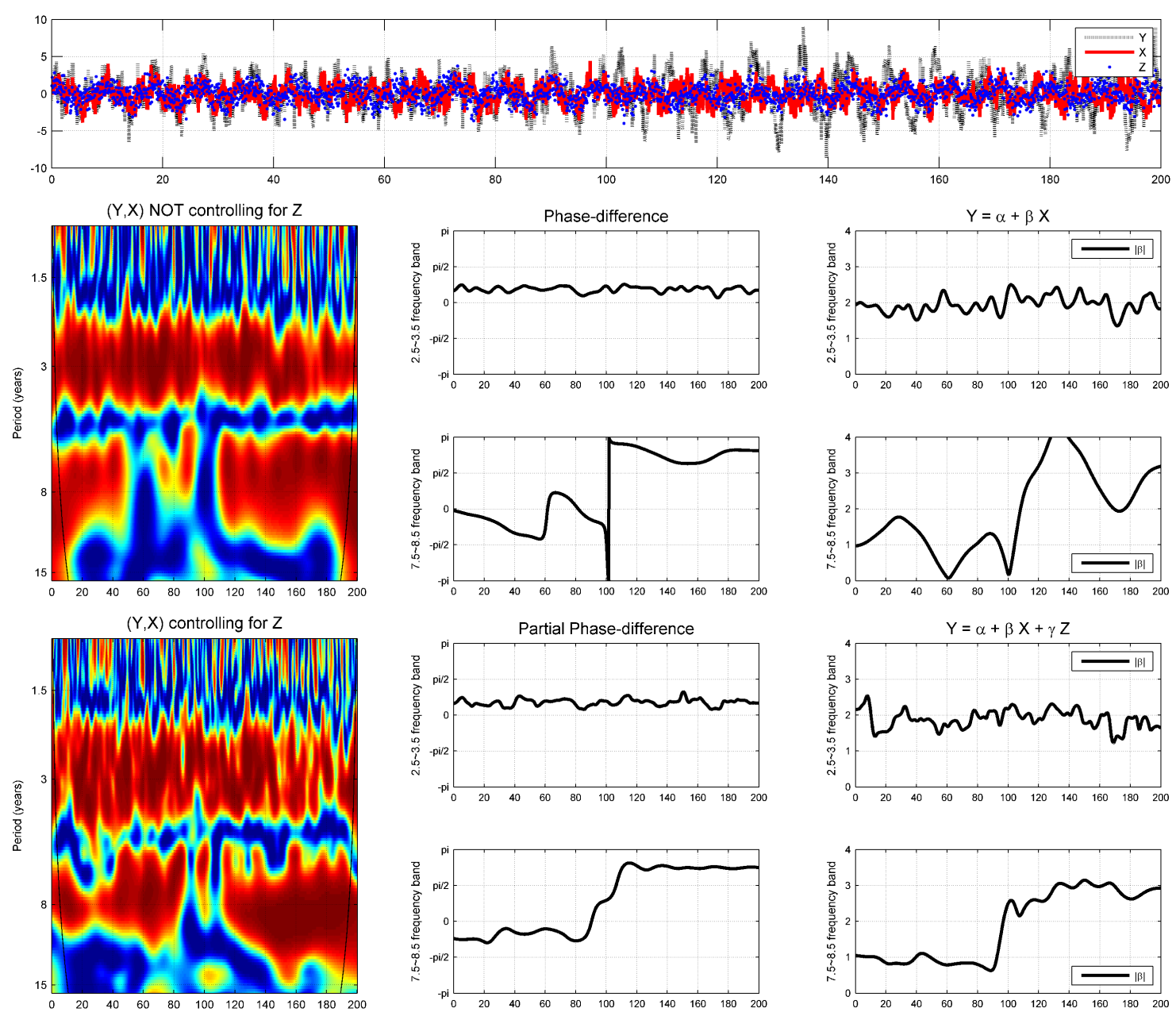

Figure 1: On the left - wavelet coherency between Y and X (top) and partial wavelet coherency between $\mathrm{Y}$ and $\mathrm{X}$, after controlling for $\mathrm{Z}$ (bottom). The color code for coherency ranges from blue (low coherency -close to zero) to red (high coherency -close to one). In the middle - phase-differences (top) and partial phase-differences (bottom) between Y and X. On the right - wavelet gain (top) and partial wavelet gain of $\mathrm{Y}$ over $\mathrm{X}$, after controlling for Z (bottom).

Figure 1 displays the results obtained with the use of the referred wavelet tools. In the left panel, we plot the wavelet coherency between $Y$ and $X$ (top) and partial wavelet coherency between $Y$ and $X$ after controlling for $Z$ (bottom). In the center panel of the figure, we present the (circular) means of the phase-differences (two top figures) and of the partial phase-differences (two bottom figures) corresponding to two different frequency bands (one for periods of 2.5 to 3.5 years and the other for periods of 7.5 to 8.5 years). On the right, we display the means of the wavelet gain of $Y$ over $X$ (two top figures) and of the partial wavelet gain of $Y$ over $X$ controlling for $Z$ (two bottom figures), corresponding to the same frequency bands.

All the expected results are confirmed in Figure 1. In particular, note how the relationships between $Y$ and $X$ around the 8-year period are more accurately estimated when we use the partial 
wavelet tool (which controls for the influence of variable $Z$ ).

\section{The Data}

Our data are quarterly time-series of the federal funds rate (FFR), inflation, and the output gap, for the U.S. 1965:IV-2017:II, which is similar to the data used by Nikolsko-Rzhevskyy, Papell, and Prodan (2014); the only difference being that it was updated through mid-2017. These data on inflation and the output gap were the real-time data available to policymakers when interest rate decisions were made; an approach that is consistent with the data selection used in most empirical research on monetary policy rules since Orphanides (2001). The source for output and inflation is the Real-Time Data Set for Macroeconomists created by Croushore and Stark (2011) and available at the Philadelphia Federal Reserve website, which provides vintages of data available since 1965:IV, with the data in each vintage starting in 1947:I. ${ }^{9}$

Inflation is the year-over-year rate of change of the real-time GDP deflator. The output gap is the percent difference between real GDP and a real-time quadratic trend, i.e., a trend obtained by fitting against a quadratic function of time, the real GDP data from 1947:I through the vintage date (see Nikolsko-Rzhevskyy, Papell, and Prodan 2014 for further details, namely on the choice of the functional form for the trend and on timing issues).

We obtained the FFR from the FRED (Federal Reserve Economic Data) database available on the website of the Federal Reserve of St. Louis, until 2008:IV and after 2015:IV. ${ }^{10}$ Between 2009:I and 2015:IV, when the policy interest rate was constrained by the zero lower bound, we used the shadow FFR of Wu and Xia (2016), which is computed from a nonlinear term structure model and captures the overall monetary policy stance, including the effects of unconventional policies. ${ }^{11}$

In Figure 2, on the left-hand side charts, we plot the three time-series, while the right-hand side shows their wavelet power spectra, which measure the variance of the series at each time-frequency locus and provide a first time-frequency description of the data. A first overall conclusion is that,

\footnotetext{
${ }^{9}$ http://www.philadelphiafed.org/research-and-data/real-time-center/real-time-data/data-files/. It should be noted that the real-time data available in this database does not strictly correspond to the Greenbook forecasts prepared by the FED staff that inform each meeting of the Federal Open Market Committee used by Orphanides (2001) and many others.

${ }^{10} \mathrm{http}: / /$ research.stlouisfed.org/fred2/.

${ }^{11}$ http://faculty.chicagobooth.edu/jing.wu/research/data/WX.html.
} 
with the exception of the 1970s instability in output and inflation, the variability of the three time-series occurs at frequencies corresponding to periods larger than 4 years. A second overall conclusion is that shorter cycles gradually lose relevance to longer cycles until the Great recession, in line with the findings of Crowley and Hallett (2015 and 2016).

The chart of inflation shows a well-known gradual rise between the mid-1960s and the 1970s, disinflation between 1980 and 1986, and low and stable inflation in the ensuing period, with particularly low rates following the recent financial and economic crisis. The wavelet power spectrum identifies three different predominant inflation cycles, whose relative importances is not constant. During the inflationary period, cycles at business-cycle frequencies ( $4 \sim 8$ years) are predominant. After that, during the disinflation period, the areas of statistically significant power spectrum become gradually thinner, which illustrates the subsequent anchoring of inflation (and its expectations) and the prolonged period of very low inflation variance during the Great Moderation.

The chart of the output gap shows the strong recession associated with the first oil shock in the mid-1970s, as well as the recession of the early 1980s associated with disinflation; it then shows the Great Moderation between 1984 and 2007, and the Great Recession starting at the end of 2007. The wavelet power spectrum indicates a prevalence of cyclical oscillations with periods below 12 years until 1985 - namely $6 \sim 8$ years and $10 \sim 12$ years —, after which shorter cycles gradually lost importance, and gave way to longer cycles along the sample. These three different cycles in the output gap were also captured by Crowley (2010), who, actually, was able to identify an even longer growth cycle (around 35 years). During the final part of the sample, cyclical variability was concentrated in cycles that had a rather long period, confirming the results of Crowley and Hughes Hallett (2014 and 2015). Our continuous time-frequency approach is also consistent with, but allows for a refinement of, the results of Pancrazi (2015), which indicated that the Great Moderation shows up as a reduction in the volatility at cycles not larger than 4 years if one assesses four frequency bands $(0.5 \sim 1.5 ; 1.5 \sim 4 ; 4 \sim 8 ; 8 \sim 20$ years $)$. 

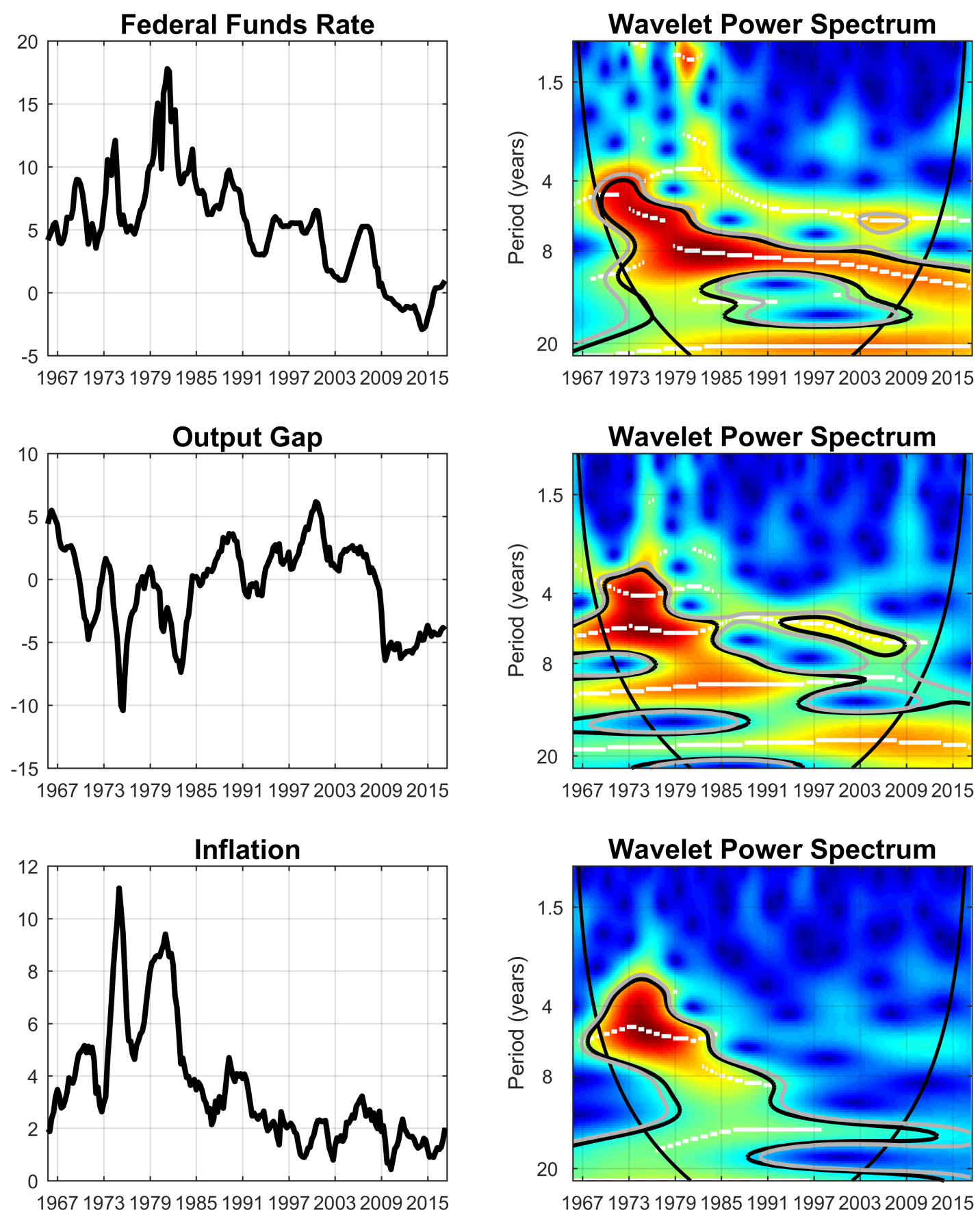

Figure 2: On the left: Plot of each time-series. On the right: The corresponding wavelet power spectrum. The black (gray) contour designates the 5\% (10\%) significance level. The cone of influence, which indicates the region affected by edge effects, is shown with a parabola-like black line. The color code for power ranges from blue (low power) to red (high power). The white lines show the local maxima of the wavelet power spectrum.

The chart of the federal funds rate (FFR) shows that nominal interest rates tended to increase with inflation since the mid-1960s, until they peaked in the beginning of the 1980s and then, as inflation got under control, gradually decreased during the remainder of the sample. The power spectrum of the FFR indicates that throughout the whole sample the variability of the policy 
rate has been systematically strong at cyclical frequencies of $8 \sim 10$ years, even though it showed particular strength during the disinflation period and, during the 1970s, at shorter cycles $(4 \sim 8$ years).

In Figure 3, we plot the Federal Funds Rate (FFR) and the Reference Policy Rule (RPR), i.e., the interest rate computed with equation (1) using our real-time output gap and inflation data. The figure conveys two main messages.

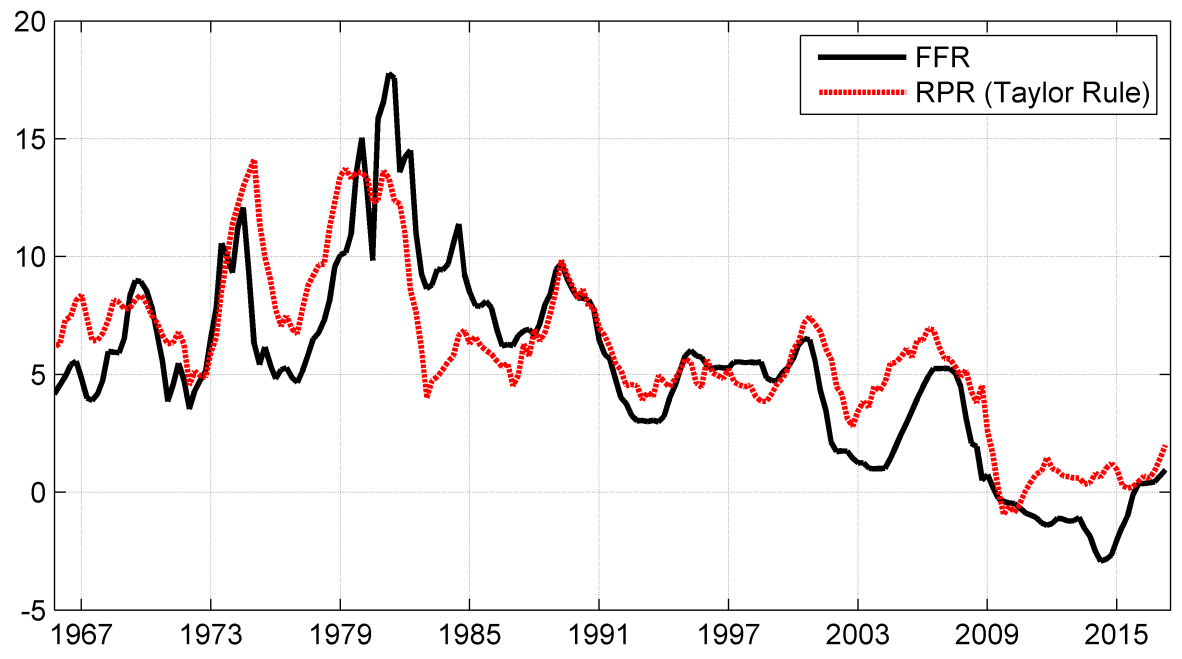

Figure 3: The Reference Policy Rule and the Federal Funds Rate since 1965 (effective for 1965:IV-2008:IV, shadow for 2009:I-2014:IV).

First, it is remarkable how the original TR broadly mimics the overall path of the policy interest rate, given its simplicity, the absence of references to interest rate rules in the Federal Open Market Committee discussions before 1993 (Kahn, 2012; Taylor, 2012), and that policymakers have never committed to a specific TR, not even a specific target (before 2012). Our finding of the overall compliance of U.S. monetary policy to the TR is confirmed by the results of an OLS regression of the TR with our real-time data for 1965:IV-2017:II (standard errors in parentheses): $\mathrm{FFT}_{t}=\underset{(0.27)}{0.16}+\underset{(0.07)}{1.54} \pi_{t}+\underset{(0.04)}{0.51} y_{t}$

The estimates for the coefficients on inflation and the output gap are almost the same as the original formulation of Taylor's Rule (the smaller intercept may be due to a higher inflation target or to a lower equilibrium real interest rate).

Second, a closer look at the figure reveals that, in many periods, the FFR looks persistently close to the RPR, while in others it deviates from RPR systematically. One approach in the literature has considered the former as episodes of rules-based policy, and the latter as ones of 
discretionary policy, typically associating better macroeconomic outcomes with the former. For example, Nikolsko-Rzhevskyy, Papell, and Prodan (2014) find that FFR followed quite closely the original Taylor Rule in 1965:IV-1974:III and in 1985:II-2001:I, deviating substantially from the rule in 1974:IV-1985:I and in 2001:II-2013:IV, with the former period split into two sub-periods: one of too-low interest rates (until 1979:IV) and another of too-high interest rates (from 1980:I to 1985:I). ${ }^{12}$ When they used a modified Taylor Rule with a coefficient of 1 on the output gap, Nikolsko-Rzhevskyy, Papell, and Prodan (2014) detected a further break and identified a period of rules-based policy in 2006:IV-2013:IV. Such modified rule is consistent with statements by the Chair of the FED pointing out that the implied interest rates are closer to those given by the optimal control solution of the FRB/US model than the interest rates implied by the original TR — see Bernanke (2011) and Yellen (2012). Indeed, it prescribes negative interest rates since 2009 — in line with the shadow FFR depicted in Figure 3 -, which the original Taylor Rule does not - also shown in the picture.

In this paper, rather than comparing the U.S. interest rate policy with the one given by the original TR, or seeking for alternative specifications of the TR with improved fit to the data, ${ }^{13}$ we give due consideration to the arguments that the policy rule coefficients may change along time and across frequencies.

\section{Results: The Taylor Rule in the Time-Frequency do- main}

We now assess the relationship between the FFR and the macroeconomic variables of the Taylor Rule in the time-frequency domain, using multivariate continuous wavelet tools. We start with the multiple coherency, ${ }^{14}$ which is the time-frequency analog of the $R^{2}$ in the typical regression. Then, we present and discuss the partial coherency, the partial phase-difference, and the partial

\footnotetext{
${ }^{12}$ Taylor (2012) and Belongia and Ireland (2016) reached similar results

${ }^{13}$ See e.g. Sims (2013), Sack and Rigobon (2003), Lubik and Schorfheide (2007), and Christensen and Nielsen (2009)

${ }^{14}$ In what follows, since we always deal with wavelet-based measures and for simplicity, we will avoid using the word wavelet and simply write multiple coherency for multiple wavelet coherency, partial gain for partial wavelet gain, etc.
} 
gain between the FFR and each of the macroeconomic variables in the Taylor Rule, controlling for the effects of the other. The latter corresponds to estimating the coefficients associated with each macro variable in the TR, allowing for their variation along time and across frequencies, i.e., estimating the Taylor Rule coefficients in the time-frequency domain. While the interpretation of our econometric results proceeds along the standard approach for the coherency and phasedifferences (see e.g. Aguiar-Conraria, Martins, and Soares, 2012), it is substantially enhanced by considering the parametric estimation of the partial gain.

Figure 4 summarizes our results. To facilitate the presentation, we provide the reader with partial phase-difference and gain diagrams displaying mean values corresponding to three frequency intervals, namely for cycles of period $1.5 \sim 4$ years (short end of business cycles), cycles of period $4 \sim 8$ years (bulk of business cycles fluctuations) and cycles of period $8 \sim 20$ years (these capture long run relationships). We note, however, that some authors, e.g. Crowley and Hughes Hallett (2015), have argued that there is a lengthening of the business cycle. Still, we stick to the traditional frequency intervals, for example, our bands are similar to Pancrazi's (2015); although we opted for a different terminology than his, as he refers to cycles of $1.5 \sim 4$ years as high business cycles, cycles of $4 \sim 8$ years as lower business cycles, and cycles of $8 \sim 20$ years as medium frequency. ${ }^{15}$

\footnotetext{
${ }^{15}$ We agree with Pancrazi (2015), who stressed that the lower frequencies of $8 \sim 20$ years display relevant information for monetary and macro analysis.
} 

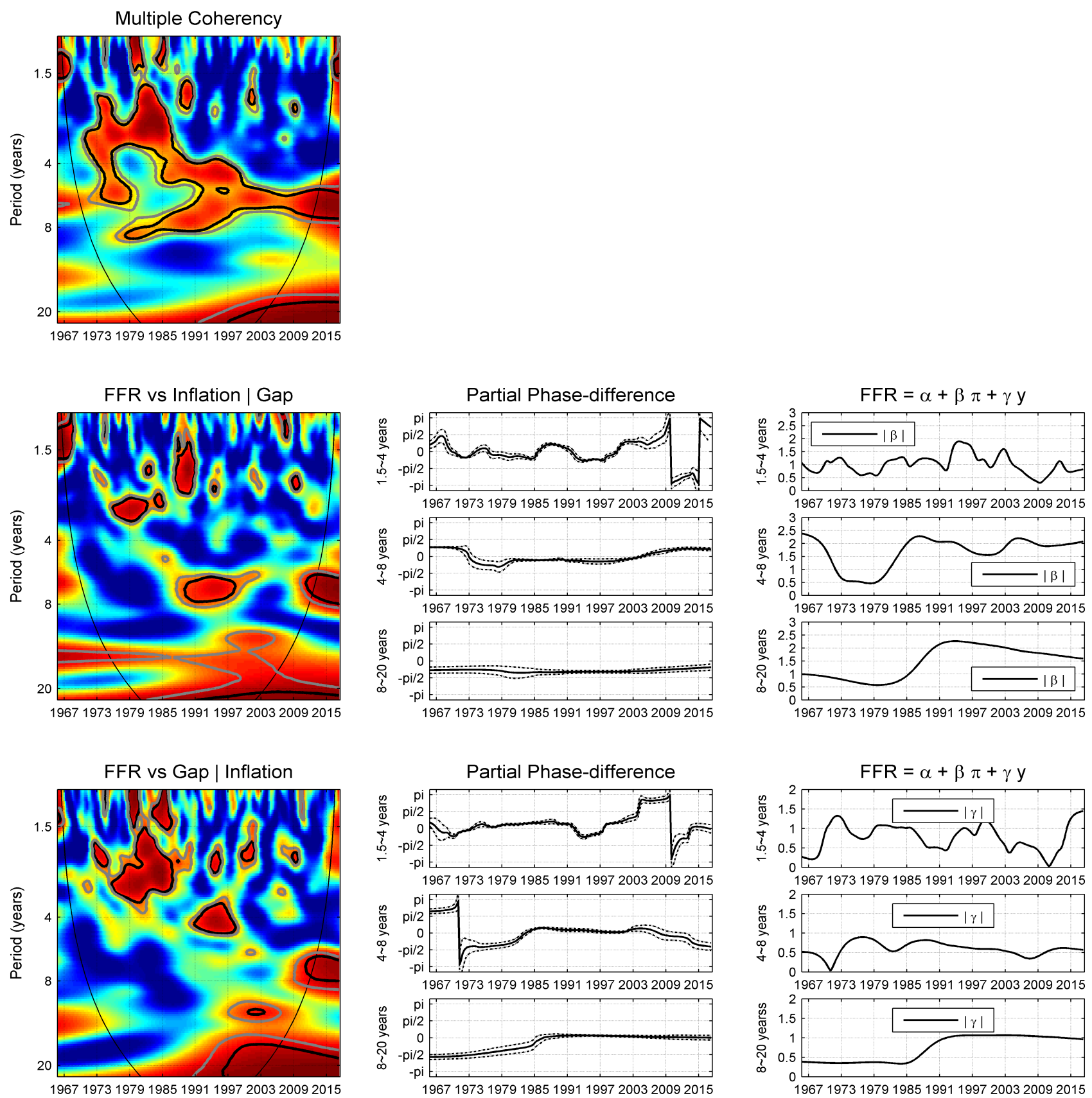

Figure 4: On the left - multiple wavelet coherency (top) and partial wavelet coherency between interest rate and inflation (middle) and between interest rate and the output gap (bottom). The black (gray) contour designates the 5\% (10\%) significance level. The color code for coherency ranges from blue (low coherency - close to zero) to red (high coherency - close to one). In the center - partial phase-differences. The limits of the confidence intervals for the mean phases are indicated in the pictures with black dashed-lines. On the right - partial wavelet gain.

FFR and the Taylor Rule For each time-frequency location, the multiple coherency measures the overall fit of the TR in the time-frequency domain: regions with a significant multiple coherency mean that inflation and the output gap are jointly significant explanatory variables of the FFR 
at those time-frequency locations. The first chart of Figure 4 confirms that the TR is, overall, a good model for the FFR, as shown by the prevalence of regions depicted with warm colors. The multiple coherency gives further time-frequency details that suggest that the overall fit of TR has gradually shifted towards cycles of longer length. During the 1970s and 1980s, multiple coherency is significant at higher frequencies $(1.5 \sim 4$ year cycles), but hardly after 1991. On the other hand, between 1985 and 2005, multiple coherency is high at typical business cycles frequencies $(4 \sim 8$ years), and after 2009 such multiple coherency is apparently intensified. At very long cycles (close to 20 years period), multiple coherency starts to increase in the 1990s, and becomes statistically significant after the beginning of the 2000s.

This pattern is consistent with the high intensity of shocks and subsequent macro and policy volatility of the 1970s, the change to a more systematic monetary policy regime and the moderation of macroeconomic volatility since 1985, the gradual conquer of credibility during the 1990s, and then the marked slowdown and the policy sharp reaction since the crisis of the late 2000s.

The multiple coherency assists in the interpretation of the results given by the partial coherencies, especially when the explanatory variables are highly related, as is the case in the TR. Our partial coherencies — to be analyzed in the next sub-sections — capture the co-movement between each explanatory variable (inflation and output gap) and the FFR, filtering out the effect of the other. Yet, there is typically a strong co-movement between inflation and the output gap, as illustrated by the Phillips Curve - indeed, the predictive power of the output gap over inflation is often invoked to motivate its inclusion in the TR. Under such circumstances, while the overall significance of the model is high, the significance of individual co-movements for both explanatory variables may appear mistakenly low. To assess this possibility, we also estimate the coherency between inflation and the output gap (not shown), which confirms that there are important regions of high coherency. It is, therefore, important that the partial coherencies be interpreted together with the multiple coherency.

A case in point is the time-frequency region between 1973 and 1980 for frequencies of $4 \sim 8$ years: while both partial coherencies are mostly blue, the multiple coherency is mostly red and statistically significant. The coherency plot between inflation and the output gap confirms that these two variables are significantly coherent in this region; hence, in spite of the apparent lack 
of statistical significance of the partial coherencies, we are able to interpret the evolution of the coefficients of inflation and the output gap in that time-frequency region.

FFR and inflation The partial coherency between FFR and inflation exhibits different patterns across our three ranges of frequency-bands. At short-run frequencies (period $1.5 \sim 4$ years), the partial coherency is strong and significant from the second half of the 1970s until the beginning of the 1990s. At typical business cycles frequencies ( $4 \sim 8$ years), coherency is strong and significant between 1985 and the end of the rules-based era, 2003, and again towards the end of the sample. At lower frequencies (period $8 \sim 20$ years), coherency is consistently strong throughout the whole period, but only significant at $10 \%$.

When coherencies are significant, the phase-differences for both the $4 \sim 8$ years and the $8 \sim 20$ years frequency bands are stable and consistently located in the interval $(-\pi / 2,0)$, indicating a positive co-movement — as expected in the TR —, with inflation leading the FFR. ${ }^{16}$ While at $4 \sim 8$ years frequencies the phase-differences are close to 0 and do not allow rejection of the hypothesis of a contemporaneous co-movement, at $8 \sim 20$ frequencies, the lag between interest rates and inflation is larger (phase-difference closer to $-\pi / 2$ ), which suggests that U.S. monetary policy has reacted more timely to inflation movements at business cycle frequencies than at longer cycles. At the $4 \sim 8$ years cycles there is a shift in the phase-differences to the interval $(0, \pi / 2)$ after 2009 , when inflation starts lagging the FFR. The phase-differences vary more in the frequency band of $1.5 \sim 4$ years, but, when coherency is significant, overall, they indicate a positive co-movement, with FFR lagging inflation until 1986 and leading inflation in 1987-91. Our analysis suggests that the reaction of interest rates to inflation has occurred with a lag at long cycles, while it has been synchronized at the typical business cycle frequencies and, after 2009, has preceded the changes in inflation - possibly reflecting the difficulties of monetary policy in stimulating inflation after the Great Recession.

We now focus on the time-frequency partial gain from FFR due to inflation, displayed in the upper three charts of the right-hand side of Figure 4. We have seen above that the full sample OLS

\footnotetext{
${ }^{16}$ It is worth noting that in the $8 \sim 20$ years frequency band the multiple coherency is low for most of the sample, therefore these results regarding partial coherency should be interpreted with some caution and may require further investigation.
} 
estimate of the slope of inflation in the TR is, essentially, Taylor's baseline value of 1.5. Looking at the time-frequency estimates, we now see that they exhibit considerable variation around that value, with important differences across our frequency bands, which indicates that the TR implicit in U.S. monetary policy has changed along both dimensions: time and frequency.

A result common to all frequency bands is that the gain is below 1.0 - violating the Taylor principle $^{17}$ — from around early 1970s to early 1980s. At higher frequencies, when significant (from the second half of the 1970s until the beginning of the 1990s), the gain fluctuates between 0.5 and 1.5. It falls below the Taylor principle threshold of 1.0 from 1974 to 1981, and then fluctuates within the range of 1.0 to 1.5 until the beginning of the $1990 \mathrm{~s}$. One should note that, since the rules-based era of 1985-2003, the inflation coefficient is systematically above the baseline vale of 1.5 both at the core of the business cycle frequencies $(4 \sim 8$ years) and at long run frequencies ( $8 \sim 20$ years). To be more precise, at the core business cycle frequencies, the gain increases from 0.5 in 1979 to 2.5 in 1987; after that its value stays between 1.5 and 2.5.

Our results are consistent with the gradual decrease of the inflation coefficient until 1979 and increase thereafter estimated by Coibion and Gorodnichenko (2011), but add important information to those studies of U.S. monetary policy that, following Clarida et al. (2000), document that U.S. policy reacted more strongly to inflation after 1979, showing that the timing and size of the change in reaction differs across cyclical frequencies.

FFR and the output gap The partial coherency between FFR and output exhibits different patterns across frequency-bands, which, to some extent, resemble the patterns of the multiple coherency and of the partial FFR-inflation coherency — a gradual shift of co-movement towards cycles of longer length. Yet, two differences are noteworthy: (1) coherency at short-run frequencies (period of $1.5 \sim 4$ years) is much more pervasive and, indeed, significant from the early 1970s until the beginning of the 2000s; and (2) coherency is much more limited at typical business cycles frequencies ( $4 \sim 8$ years), at which it is only significant during the 1990 s and after 2009 , this latter

\footnotetext{
${ }^{17}$ In this paper, "Taylor principle" means a coefficient of inflation above 1 . This is a pragmatic reference to the principle that is often used in the empirical literature on the TR, indicating whether the policy interest rate is changed more or less than proportionally with inflation and whether monetary policy is prima facie stabilizing or un-stabilizing. It should be noted, however, that the "Taylor principle" is a theoretical concept and, as such, model dependent.
} 
time frame is characterized by highly expansionary policy to fight the Great Recession.

When coherencies are significant, the phase-differences consistently indicate a positive comovement between the FFR and the output gap, as expected, with FFR slightly leading output. There are two major exceptions: (1) in the early 1990s' downturn — specifically between 1993 and 1998 - for short cycles (1.5 4 years) and (2) after 2009, at the typical business cycles ( $4^{\sim} 8$ years); in both cases the phase-differences are located in the $(-\pi / 2,0)$, meaning that the output gap was leading the FFR. Overall (with the exception of those two very precise time-frequency episodes), the partial coherencies and phase-differences indicate that U.S. monetary policy has attempted to preemptively stabilize the output gap for most of the time span and frequency bands during which the FFR and the output gap have co-moved significantly; such time difference exhibited in the positive co-movement in the data is consistent with the lags in the transmission of interest rate policy to real activity (and inflation). In turn, the partial-coherencies and phase-differences for 1993-98 (1.5 $\sim 4$ years cycles) and post-2009 ( $4 \sim 8$ years cycles $)$ are consistent with episodes of very abrupt and marked cyclical downturns that monetary policy could not duly anticipate and tame.

To obtain quantifiable results of the coefficient of output for the U.S. TR, we now assess the time-frequency partial gain from FFR to the output gap, as displayed in the three charts on the bottom right-hand side of Figure 5.

At short-run frequencies (period of $1.5 \sim 4$ years), during the extended period in which it is consistently significant (from the early 1970s until the beginning of the 2000s), the gain is close to 1.0 most of the time, with the only noteworthy exception being in 1989-1993, when it falls to the baseline value of 0.5 .

At business cycles frequencies $(4 \sim 8$ years $)$, in the first episode in which it is significant (1990s), the gain starts at a value close to 1.0 in 1990 and gradually falls until 1997 to the baseline value of 0.5 . In the second period of statistical significance (2009 onwards), the gain increases somewhat, reaching values above 0.5, which subsist until the end of the sample. Previously, since the early 1970 s — when the partial coherency is not significant, but the multiple coherency is the gain fluctuates between the full sample estimate of 0.5 and the value of 1.0 featured at the beginning of the 1990s. 
At frequencies corresponding to the $8 \sim 20$ years period, when statistically significant - i.e. after early $1990 \mathrm{~s}^{18}$-, the gain is consistently close to 1.0 , a level that it maintains until the end of the sample period. Previously, when coherency started to increase, although not achieving statistically significance — second half of the 1980s — the gain increased from a value slightly below 0.5 , reaching the level of 1.0 by the beginning of the $1990 \mathrm{~s}$.

Our estimates for the gains of the output gap across frequencies, hence, suggest that the full sample OLS estimate of 0.5 is an artifact resulting from different coefficients across frequencies and over time. Before the beginning of the 1990s, values of the gain below 0.5 at the $8 \sim 20$ cycles are offset by values mostly above 0.5 at the $4 \sim 8$ years and $1.5 \sim 4$ years cycles; after 1991, values of the gain close to 1.0 at the $8 \sim 20$ cycles are compensated by values gradually smaller and close to 0.5 after 1997 at the $4 \sim 8$ cycles, and after 2003 at the $1.5 \sim 4$ years cycles. In the brief episode of the early-1990s, in which the gain is above 0.5 at both the $4 \sim 8$ and $8 \sim 20$ years frequency bands, it is particularly low at the $1.5 \sim 4$ years cycles - actually, around 0.5 between 1989 and 1993.

In the latter part of the sample, our estimates for the gains show that findings elsewhere in the literature and statements by policy-makers pointing to a coefficient of 1.0 on the output gap in the U.S. TR since the Great Recession is not evenly explained across frequencies. After 2009, the estimate for the gain at the $8 \sim 20$ years cycles band is consistently very close to 1.0 , the gain at the $1.5 \sim 4$ years frequency band sharply increases from 0 to 1.5 , while the gain at the $4 \sim 8$ years cycles is close to 0.5. With time-series tools, Nikolsko-Rzhevskyy, Papell, and Prodan (2014) found that, since 2007, U.S. monetary policy follows a modified Taylor Rule with a coefficient of 1.0 on the output gap; a coefficient twice as large as that in the original TR is consistent with the preferences for a balanced approach that stabilizes output and prices, as stated by Federal Reserve Chairs during the Great Recession — see Bernanke (2011) and Yellen (2012); moreover, it is consistent with negative policy interest rates since 2009, in line with the estimated shadow FFR for that period - which the original TR, with a 0.5 coefficient on the output gap, is not.

\footnotetext{
${ }^{18}$ The fact that we find a statistically significant co-movement between FFR and the output gap is not completely surprising. For example, Verona (2016), while studying the financial cycle, found that GDP, house prices, and total credit had important cycles of long duration, 12 to 20 year periods. However, Verona did not estimate the coherence between these variables, so it is difficult to speculate further regarding how compatible his results are with ours.
} 
Our framework shows that the prevalence of a modified TR with a slope of 1.0 on the output gap since 2009 is associated to policy actions focusing on the long end ( $8 \sim 20$ years) of cyclical oscillations, rather than on the most standard business cycle frequencies.

\section{Conclusions}

The work of Crowley (2007), with an excellent review of economic applications of the Discrete Wavelet Transform, but also with a subsection on the continuous transform, stimulated a growing literature on economic applications of wavelet tools. For recent developments the reader is invited do read Aguiar-Conraria and Soares (2014) and the chapters included in the book edited by Gallegati and Semmler (2014). From our point of view, as macroeconomists, in the future we expect these tools to be used to test DSGE models. Watson (1993) — see also Wen (1998) - proposes the use of spectral measures to test business cycle models. Wavelet measures, in principle, could be applied in the same vein, with the advantage of also being used to test model fit for particular historical episodes. This is so because, contrary to spectral analysis, with wavelet analysis one does not lose the time dimension. Our paper fits well in this growing literature both from a methodological and from an empirical point of view.

We have argued that the partial wavelet gain could be used as an estimation of a multivariate regression in the time-frequency space. That means that we are able to estimate not only time-varying coefficients, but also frequency varying coefficients in a direct fashion. To correctly interpret the partial wavelet gain, one must also make use of the other typical wavelet tools such as the wavelet partial coherency and the phase-difference. While coherency is necessary to ascertain the statistical significance of the estimated relationships, the phase-difference is essential to inform us on whether the estimated relationships are positive or negative. This is so because, by definition, the (partial) wavelet gain is an absolute value of a complex number and, therefore, we lose the plus or minus sign characteristic of a typical regression coefficient. With the partial wavelet gain, we do not have the possibility of simultaneously including contemporaneous and lagged values of the same variable, or include as a regressor the lagged value of the dependent variable, which makes the partial phase-difference an important source of information about the 
leads and lags between the different time series.

We applied these techniques to assess U.S. monetary policy in 1965:IV-2017:II across time and frequencies in the framework of the Taylor Rule (TR). While changes in the TR coefficients along time have already been the subject of a vast literature, the variations of the TR coefficients simultaneously in the time and frequency domains were yet to be studied. We were able to reach several conclusions.

The first thing to note, which is clear from our multiple coherency measures, is that TR is, as a whole, a good model for the FFR, but the fit evolves both across time and frequencies, gradually shifting towards cycles of longer length throughout most of the five decades, but then featuring a regain of relevance of the typical business cycles after the Great Recession. The overall result for the bulk of the five decades is consistent with the work of Crowley and Hughes Hallett (2014, 2015, and 2016), while the findings for the post-2009 period at the business cycle frequencies are novel. Crowley and Hughes Hallet (2015 and 2016) relied on the discrete wavelet transform to decompose GDP and its components into different time-scales and concluded that the great moderation was more of a transfer of volatility from high to low frequencies, rather than an actual moderation. Moreover, Crowley and Hughes Hallet (2014) used a new Keynesian model (which, naturally, included a version of the Taylor rule) to argue that this can be the result of an increase in inflation aversion or a reduction of the commitment to output stabilization. Our results suggest that the former explanation is more likely to be correct than the latter.

As for the relationship between inflation and FFR, we were able to observe a consistently positive relationship at all frequencies. At business cycle frequencies the phase-difference is close to zero most of the time, informing us that these variables move together and at the same time. The period after 2009 is an exception, with the phase-difference suggesting that inflation lags the FFR, consistently with the difficulties of monetary policy to re-inflate the economy after the Great Recession. At lower frequencies (cycles of $8 \sim 20$ years), the interest rate lags inflation, which suggests that, for longer cycles, monetary policy has reacted more slowly to developments in inflation, possibly benefiting from the credibility acquired with strong policy stances at the business cycle frequencies. Regarding the estimated coefficients, we note that for frequencies that represent the bulk of business cycles fluctuations, the coefficient is consistently above the 1.5 
benchmark, except between 1973 and 1979, when it fell to 0.5, increasing after that to values between 2 and 2.5 after 1985. At lower frequencies ( $8 \sim 20$ years), the coefficient dropped from almost 1 in the beginning of the sample to 0.5 in 1979, after which it increased to an estimate somewhat above 2 in 1991, then decreasing consistently to an estimate of 1.5 at the end of the sample. These results indicate that, in the 1980s, there was a clear shift in monetary policy toward a strong preference for keeping inflation stable, which survives until today.

Concerning the output gap, we document a gradual shift of the co-movement between the FFR and the output gap towards cycles of longer length, along five decades of U.S. monetary policy. We confirm that the co-movement between the FFR and output has been positive at all frequencies, with the policy rate slightly leading output for most of the time and frequencies, which is consistent with an anti-cyclical stance and with the lags of policy impact. Regarding the coefficient estimates, we document that the full sample OLS estimate of 0.5 is an artifact resulting from different coefficients across frequencies and along time. For example, estimates close to 1, at the $8 \sim 20$ years cycles since the 1990s, are offset by estimates closer to 0.5 at the $4 \sim 8$ year cycles in that period. Moreover, we show that neither time-series evidence, nor policymakers' statements pointing to a modified TR with a slope of 1.0 on the output gap in the U.S. TR since the Great Recession, are evenly explained across frequencies; instead we find support for stronger reactions of policy to the output gap to be more commonly associated to the long-end ( $8 \sim 20$ years) of cyclical oscillations, but not to the most standard $(4 \sim 8$ years) business cycle frequencies.

\section{References}

[1] Aguiar-Conraria, Luís, Manuel M. F. Martins, and Maria J. Soares (2012) "The Yield Curve and the Macro-Economy Across Time and Frequencies" Journal of Economic Dynamics and Control, 36, 1950-1970.

[2] Aguiar-Conraria, Luís, and Maria J. Soares (2014) "The Continuous Wavelet Transform: Moving beyond Uni-and Bivariate Analysis" Journal of Economic Surveys, 28, 344-375.

[3] Ahmed, Shaghil, Andrew Levin, and Beth Anne Wilson (2004) "Recent U.S. Macroeconomic Stability: Good Policies, Good Practices, or Good Luck?" The Review of Economics and Statistics, 86, 824-832.

[4] Alcidi, Cinzia, Alessandro Flamini, and Andrea Fracasso (2011) "Policy Regime Changes, Judgment and Taylor Rules in the Greenspan Era" Economica, 78, 89-107. 
[5] Ashley, Richard, Kwok Ping Tsang, Randal J. Verbrugge (2015) "Frequency Dependence in a Real-Time Monetary Policy Rule." Virginia Polytechnic Institute and State University Working Paper e07-43 (revised: September 23, 2015).

[6] Assenmacher-Wesche, Katrin (2006). "Estimating Central Banks' Preferences from a Timevarying Empirical Reaction Function." European Economic Review, 50, 1951-1974.

[7] Belongia, Michael T., and Peter N. Ireland (2016) "The Evolution of US Monetary Policy: 2000-2007." Journal of Economic Dynamics and Control, 73, 78-93.

[8] Bernanke, Ben S. (2011) "Witness before the Committee on Banking, Housing, and Urban Affairs of the U. S. Senate", March 1, available at http://www.gpo.gov/fdsys/pkg/CHRG112shrg65824/pdf/CHRG-112shrg65824.pdf.

[9] Bernanke, Ben S. (2015) "The Taylor Rule: A benchmark for monetary policy?", Ben Bernanke's Blog, April 28, 2015 11:00am, available at http://www.brookings.edu/blogs/benbernanke/posts/2015/04/28-taylor-rule-monetary-policy.

[10] Berens, Philipp (2009) "CircStat: A MATLAB Toolbox for Circular Statistics." Journal of Statistical Software, 31 (10), 1-21.

[11] Bunzel, Helle, and Walter Enders (2010) "The Taylor Rule and "Opportunistic" Monetary Policy." Journal of Money, Credit and Banking, 42, 931-949.

[12] Christensen, Anders M., and Heino B. Nielsen (2009) "Monetary Policy in the Greenspan Era: A Time Series Analysis of Rules vs. Discretion." Oxford Bulletin of Economics and Statistics, $7(1), 69-89$.

[13] Clarida, Richard, Jordi Galí, and Mark Gertler (2000) "Monetary Policy Rules and Macroeconomic Stability: Evidence and Some Theory." Quarterly Journal of Economics, 115, 147-80.

[14] Coibion, Olivier, and Yuriy Gorodnichenko (2011) "Monetary Policy, Trend Inflation and the Great Moderation: An Alternative Interpretation." American Economic Review, 101, 341-370.

[15] Croushore, Dean and Tom Stark (2011) "A real-time data set for macroeconomists." Journal of Econometrics, 105 (1), 111-130.

[16] Crowley, Patrick M. (2007), "A guide to wavelets for economists", Journal of Economic Surveys, 21 (2), 207-267.

[17] Crowley, Patrick M. (2010), "Long Cycles in Growth: Explorations Using New Frequency Domain Techniques with US Data", Bank of Finland Research Discussion Paper No. 6/2010.

[18] Crowley, Patrick M. and Andrew Hughes Hallett (2014) "Volatility transfers between cycles: A theory of why the "great moderation" was more mirage than moderation." Bank of Finland Research Discussion Papers 23.

[19] Crowley, Patrick M. and Andrew Hughes Hallett (2015) "Great moderation or "Will o' the Wisp"? A time-frequency decomposition of GDP for the US and UK." Journal of Macroeconomics 44, 82-97. 
[20] Crowley, Patrick M. and Andrew Hughes Hallett (2016) "Correlations Between Macroeconomic Cycles in the US and UK: What Can a Frequency Domain Analysis Tell Us?" Italian Economic Journal, 2, 5-29.

[21] Engle, Robert (1976) "Interpreting spectral analyses in terms of time-domain models." Annals of Economics and Social Measurement, 5(1), 89-109.

[22] Gallegati, Marco and Willi Semmler (2014) Wavelet Applications in Economics and Finance, Springer International Publishing (2014)

[23] Hamilton, James D. (2001). "A Parametric Approach to Flexible Nonlinear Inference." Econometrica, 69, 537-573.

[24] Kahn, Geroge A. (2012) "The Taylor Rule and the Practice of Central Banking," in The Taylor Rule and the Transformation of Monetary Policy, edited by Evan F. Koenig, Robert Leeson, and George A. Kahn, Chapter 3, Hoover Institution, Stanford University.

[25] Kim, Dong H., Denise Osborn, and Marianne Sensier (2005) "Nonlinearity in the Fed's Monetary Policy Rule." Journal of Applied Econometrics, 20, 621-639.

[26] Lee, Kevin, James Morley, and Kalvinder Shields (2015) "The meta Taylor rule." Journal of Money, Credit and Banking, 47 (1), 73-98.

[27] Lubik, Thomas A. and Frank Schorfheide (2007) "Do Central Banks Respond to Exchange Rate Movements? A Structural Investigation." Journal of Monetary Economics, 54, 10691087.

[28] Mandler, Martin, and Michael Scharnagl (2014) "Money growth and consumer price inflation in the euro area: a wavelet analysis." Discussion Paper, Deutsche Bundesbank No 33/2014.

[29] Mehra, Y. P., and Sawhney, B. (2010) "Inflation measure, Taylor rules, and the GreenspanBernanke years." Federal Reserve Bank of Richmond Economic Quarterly, 96 (2), 123-151.

[30] Nikolsko-Rzhevskyy, Alex, David H. Papell, and Ruxandra Prodan (2014). "Deviations from rules-based policy and their effects." Journal of Economic Dynamics and Control , 49, 4-17.

[31] Orphanides, Athanasios (2001) "Monetary Policy Rules Based on Real-time Data." American Economic Review, 91, 964-985.

[32] Pancrazi, Roberto (2015) "The Heterogenous Great Moderation." European Economic Review, 74, February, 207-228.

[33] Sack, Brian, and Roberto Rigobon (2003) "Measuring the Reaction of Monetary Policy to the Stock Market." Quarterly Journal of Economics, 118, 639-669.

[34] Sims, Eric (2013) "What Measure of Output Should be Targeted in Interest Rate Rules?" Mimeo, March 7, 2013. Available at http://brassmonkey.tamu.edu/common/files/papers/Sims-Eric.pdf

[35] Taylor, John B. (1993) "Discretion Versus Policy Rules in Practice." Carnegie-Rochester Conference Series on Public Policy, 39, 195-214. 
[36] Taylor, John B. (2012) "Monetary Policy Rules Work and Discretion Doesn't: A Tale of Two Eras." Journal of Money, Credit and Banking, 44, 1017-1032.

[37] Taylor, John B., and John C. Williams. (2010) "Simple and Robust Rules for Monetary Policy." In Handbook of Monetary Economics, Volume 3, edited by Benjamin M. Friedman and Michael Woodford, pp. 829-859. Elsevier.

[38] Torrence, Cristopher and Compo, Gilbert P. (1998). "A practical guide to wavelet analysis." Bulletin of the American Meteorological Society 79: 61-78.

[39] Trecroci, Carmine, and Matilde Vassalli (2010) "Monetary Policy Regime Shifts: New Evidence from Time-Varying Interest Rate Rules." Economic Inquiry, 48, 933-950.

[40] Verona, Fabio (2016) "Time-frequency characterization of the U.S. financial cycle." Economics Letters, 144, 75-79.

[41] Walsh, Carl E. (2015) "Goals and Rules in Central Bank Design." International Journal of Central Banking, 11 (S.01), 295-352.

[42] Watson, mark (1993) "Measures of fit for calibrated models." Journal of Political Economy, 101 (6), 1011-1041.

[43] Wen, Yi (1998) "Can a real business cycle model pass the Watson test?" Journal of Monetary Economics, 42(1), 185-203

[44] Wolters, Maik H. (2012) "Estimating Monetary Policy Reaction Functions Using Quantile Regressions." Journal of Macroeconomics, 34, 342-361.

[45] Wu, Jing Cynthia, and Fan Dora Xia (2016) "Measuring the macroeconomic impact of monetary policy at the zero lower bound." Journal of Money, Credit and Banking, 48, 253-291.

[46] Yellen, Janet (2012) "The Economic Outlook and Monetary Policy", Remarks at the Money Marketeers of New York University, April 11, available at http://www.federalreserve.gov/newsevents/speech/yellen20120411a.pdf.

[47] Yu, Xiangrong. (2013) "Measurement Error and Policy Evaluation in the Frequency Domain." Journal of Macroeconomics, 38, 307-329.

[48] Zar, Jerrold H. (1996) Biostatistical Analysis (3rd Ed.), Prentice-Hall. Upper Saddle River, New Jersey. 


\section{A Methodological Appendix - Multivariate Wavelet Analy- sis, the General Case}

For the benefit of the reader, we first introduce our notation. Given $p$ time-series, $x_{1}, x_{2}, \ldots, x_{p}$, with $p>2$, we will denote by $W_{i}$ the wavelet spectrum corresponding to the $i$-th time-series and by $W_{i j}$ the cross-wavelet spectrum of two series, $x_{i}$ and $x_{j}$, such that $1 \leqslant i \leqslant p, 1 \leqslant j \leqslant p$. Similarly to ordinary wavelet coherency, one must perform a smoothing operation of the crossspectra to compute partial wavelet coherencies. We denote by $S_{i j}$ the smoothed version of $W_{i j}$, i.e. $S_{i j}=S\left(W_{i j}\right)$, where $S$ is a certain smoothing operator and will use $\mathscr{S}$ to denote the $p \times p$ matrix of all the smoothed cross-wavelet spectra $S_{i j}$, i.e., $\mathscr{S}=\left(S_{i j}\right)_{i, j=1}^{p} \cdot{ }^{19}$ For a given matrix $A, A_{i}^{j}$ denotes the sub-matrix obtained by deleting its $i$-th row and $j$-th column and $A_{i j}^{d}$ denotes the co-factor of the element in position $(i, j)$ of $A$, i.e. $A_{i j}^{d}=(-1)^{(i+j)} \operatorname{det} A_{i}^{j}$. For completeness, we use the notation $A^{d}=\operatorname{det} A$. Finally, for a given integer $j$ such that $2 \leq j \leq p$, we denote by $q_{j}$ the set of all the indexes from 2 to $p$ with the exception of $j$, i.e. $q_{j}=\{2, \ldots, p\} \backslash\{j\}$.

\section{A.1 Multiple and partial wavelet coherency and partial phase-difference}

The squared multiple wavelet coherency between the series $x_{1}$ and all the other series $x_{2}, \ldots, x_{p}$ is denoted by $R_{1(23 \ldots p)}^{2}$ and given by the formula

$$
R_{1(23 \ldots p)}^{2}=1-\frac{\mathscr{S}^{d}}{S_{11} \mathscr{S}_{11}^{d}}
$$

The complex partial wavelet coherency of $x_{1}$ and $x_{j}(2 \leq j \leq p)$ allowing for all the other series is denoted by $\varrho_{1 j . q_{j}}$ and given by

$$
\varrho_{1 j . q_{j}}=-\frac{\mathscr{S}_{j 1}^{d}}{\sqrt{\mathscr{S}_{11}^{d}} \sqrt{\mathscr{S}_{j j}^{d}}} .
$$

The partial wavelet coherency of $x_{1}$ and $x_{j}$ allowing for all the other series, denoted by $R_{1} j_{j}$, is defined as the absolute value of the above quantity, i.e. $R_{1 j . q_{j}}=\frac{\left|\mathscr{S}_{j 1}^{d}\right|}{\sqrt{\mathscr{S}_{11}^{d}} \sqrt{\mathscr{S}_{j j}^{d}}}$, and the squared partial wavelet coherency of $x_{1}$ and $x_{j}$ allowing for all the other series, is simply the square of

\footnotetext{
${ }^{19}$ To be more accurate, $\mathscr{S}$ depends on the specific value $(\mathrm{t}, \mathrm{s})$ at which the spectra are being computed, i.e., there is one such matrix for each $(t, s)$.
} 
$R_{1 j . q_{j}}$. Having defined the complex partial wavelet coherency $\varrho_{1 j . q_{j}}$ of series $x_{1}$ and $x_{j}$ controlling for all the other series, we simply define the partial phase-difference of $x_{1}$ and $x_{j}$ given for all the other series, denoted by $\phi_{1 j \cdot q_{j}}$, as the angle of $\varrho_{1 j \cdot q_{j}}$.

\section{A.2 Partial wavelet gain}

We define the complex partial wavelet gain of series $x_{1}$ over series $x_{j}$ allowing for all the other series, denoted by $\mathcal{G}_{1}{ }_{j . q_{j}}$, by the formula

$$
\mathcal{G}_{1 j . q_{j}}=-\frac{\mathscr{S}_{j 1}^{d}}{\mathscr{S}_{11}^{d}}
$$

and the partial wavelet gain, denoted by $G_{1 j . q_{j}}$, as the modulus of the above quantity, i.e.,

$$
G_{1 j . q_{j}}=\frac{\left|\mathscr{S}_{j 1}^{d}\right|}{\mathscr{S}_{11}^{d}}
$$

Naturally, the complex partial wavelet gain can also be computed using the complex partial wavelet coherency, as

$$
\mathcal{G}_{1 j . q_{j}}=\varrho_{1 j \cdot q_{j}} \frac{\sqrt{\mathscr{S}_{j j}^{d}}}{\sqrt{\mathscr{S}_{11}^{d}}}
$$

and the partial wavelet gain as

$$
G_{1 j . q_{j}}=R_{1 j . q_{j}} \frac{\sqrt{\mathscr{S}_{j j}^{d}}}{\sqrt{\mathscr{S}_{11}^{d}}} .
$$

For $j=2, \ldots, p$, the values $G_{1 j . q_{j}}$ can be interpreted as the (modulus) of the coefficients in the multiple linear regression of $x_{1}$ against the explanatory variables $x_{2}, \ldots, x_{p}$, at each time and frequency.

\section{A.3 Formulas in terms of coherencies}

The above formulas for the multiple coherency, partial wavelet coherencies and partial wavelet gains were given in terms of the smoothed spectra, $S_{i j}$. We can also define these quantities in terms of simple complex coherencies (i.e., wavelet complex coherencies between pairs of series). 
Corresponding to the matrix $\mathscr{S}$, we now consider matrix $\mathscr{C}=\left(\varrho_{i j}\right)_{i, j=1}^{p}$ of all the complex wavelet coherencies $\varrho_{i j}$. Then, we can define the multiple wavelet coherency by the following alternative formula

$$
R_{1(23 \ldots p)}^{2}=1-\frac{\mathscr{C}^{d}}{\mathscr{C}_{11}^{d}}
$$

the complex partial wavelet coherency $\varrho_{1 j \cdot q_{j}}$ and the wavelet coherency $R_{1 j . q_{j}}$ by

$$
\varrho_{1 j . q_{j}}=-\frac{\mathscr{C}_{j 1}^{d}}{\sqrt{\mathscr{C}_{11}^{d}} \sqrt{\mathscr{C}_{j j}^{d}}} \quad \text { and } \quad R_{1 j . q_{j}}=\frac{\left|\mathscr{C}_{j 1}^{d}\right|}{\sqrt{\mathscr{C}_{11}^{d}} \sqrt{\mathscr{C}_{j j}^{d}}}
$$

respectively, and the complex partial wavelet gain $\mathcal{G}_{1 j . q_{j}}$ and the partial wavelet gain $G_{1 j \cdot q_{j}}$ by

$$
\mathcal{G}_{1 j . q_{j}}=-\frac{\mathscr{C}_{j 1}^{d}}{\mathscr{C}_{11}^{d}} \frac{\sigma_{1}}{\sigma_{j}} \quad \text { and } \quad G_{1 j . q_{j}}=\frac{\left|\mathscr{C}_{j 1}^{d}\right|}{\mathscr{C}_{11}^{d}} \frac{\sigma_{1}}{\sigma_{j}}
$$

respectively.

The proof of the above results is very simple: recalling that $S_{i j}=\sigma_{i} \sigma_{j} \varrho_{i j}$ (cf. (6)) and using the well-known fact that, if a row or a column of a square matrix is multiplied by a constant, the corresponding determinant is multiplied by that constant, we immediately obtain the following relations:

$$
\begin{aligned}
\mathscr{S}^{d} & =\sigma_{1}^{2} \sigma_{2}^{2} \ldots \sigma_{p}^{2} \mathscr{C}^{d} \\
\mathscr{S}_{1 j}^{d} & =\sigma_{1} \sigma_{2}^{2} \ldots \sigma_{j-1}^{2} \sigma_{j} \sigma_{j+1}^{2} \ldots \sigma_{p}^{2} \mathscr{C}_{1 j}^{d} \quad(1<j \leq p)
\end{aligned}
$$

and

$$
\mathscr{S}_{j j}^{d}=\sigma_{1}^{2} \sigma_{2}^{2} \ldots \sigma_{j-1}^{2} \sigma_{j+1}^{2} \ldots \sigma_{p}^{2} \mathscr{C}_{j j}^{d} \quad(1 \leq j \leq p)
$$

Appropriate replacement of the above relationships in formulas (A.1)- (A.4) give us formulas $(\mathrm{A} .7)-(\mathrm{A} .9)$, respectively. 